\title{
Program on high value cost-conscious education in Intensive Care: Educational program on prediction of outcome and cost awareness on Intensive Care admission
}

\section{CURRENT STATUS: UNDER REVIEW}

BMC Medical Education BMC series

Linda van Wagenberg

Universitair Medisch Centrum Utrecht

- I.vanwagenberg@umcutrecht.nICorresponding Author

ORCiD: https://orcid.org/0000-0003-0702-6265

Charlotte J. P. Beurskens

Amsterdam Universitair Medische Centra

Inge Stegeman

UMC Utrecht Brain Center Rudolf Magnus

Marcella C. A. Müller

Amsterdam Universitair Medische Centra

\section{DOI:}

$10.21203 /$ rs.2.20045/v1

\section{SUBJECT AREAS}

Internal Medicine Educational Philosophy and Theory

\section{KEYWORDS}

High-value cost-conscious care, post-graduate education, curriculum development, evaluation of education 
Abstract

Introduction: Intensive Care (ICU) involves extended and long lasting support of vital functions and organs. However, current training programs of ICU residents mainly focus on extended support of vital functions and barely involve training on cost-awareness and outcome. We incorporated an educational program on high-value cost-conscious care for residents and fellows on our ICU and measured the effect of education. Methods: A cohort study with factorial survey design, in which residents and fellows ICU were asked to evaluate clinical vignettes, was performed on the mixed surgical-medical ICU of the Amsterdam University Medical Centre. Residents were offered an educational program focusing on outcome and costs of ICU care. Before and after the program they filled out a questionnaire, which consisted of 23 vignettes, in which known predictors of outcome of community acquired pneumonia (CAP), pancreatitis, acute respiratory distress syndrome (ARDS) and cardiac arrest were presented, together with varying patient factors (age, body mass index (BMI), acute kidney failure (AKI) and haemato-oncological malignancy). Participants were asked to either admit the patient or estimate mortality. Results: BMI, haemato-oncological malignancy and severity of pancreatitis were discriminative for admission to ICU in clinical vignettes on pancreatitis and CAP. After education, only severity of pancreatitis was judged as discriminative. Before the intervention only location of cardiac arrest (in- vs out of hospital) was distinctive for mortality, afterwards this changed to presence of haemato-oncological malignancy. Conclusion: We incorporated an educational program on high-value cost-conscious care in the training of ICU physicians. Based on our vignette study, we conclude that the improvement of knowledge of costs and prognosis after this program was limited.

\section{Full Text}

Due to technical limitations, full-text HTML conversion of this manuscript could not be completed. However, the manuscript can be downloaded and accessed as a PDF.

\section{Tables}




\begin{tabular}{|c|c|c|}
\hline \multirow[t]{4}{*}{ All cases } & Presence of haematological malignancy & Yes/no \\
\hline & Acute kidney failure & Yes/no \\
\hline & Age & $<60$ years $/ 60-80$ years $/>80$ yt \\
\hline & $\mathrm{BMI}$ & $<20 / 20-25 />25$ \\
\hline \multirow[t]{2}{*}{ Community acquired pneumonia } & Presence of COPD & Absent / mild / severe \\
\hline & $\begin{array}{l}\text { Severity of Pneumonia (measured CURB } \\
-65 \text { score) }\end{array}$ & CURB-65 score 0-1 / 2 / 3-5 \\
\hline \multirow[t]{2}{*}{ Acute Pancreatitis } & Presence of chronic liver disease & Absent/ Liver cirrhosis Child Pug \\
\hline & $\begin{array}{l}\text { Severity of pancreatitis, measured by } \\
\text { Ranson score }\end{array}$ & Ranson Score 3-4/ 5-6/ 7-8 \\
\hline \multirow[t]{2}{*}{ ARDS } & Type of ARDS & Primary/ Secondary \\
\hline & Severity of ARDS & None/ Mild/ Moderate/ Severe \\
\hline \multirow[t]{3}{*}{ Cardiac arrest } & First rhythm & $\begin{array}{l}\text { Shockable (VF/ VT)/ Non-shocka } \\
\text { Asystole) }\end{array}$ \\
\hline & Location of arrest & $\begin{array}{l}\text { Out of hospital cardiac arrest/ In } \\
\text { cardiac arrest }\end{array}$ \\
\hline & Delay until start CPR & $<3$ minutes $/>3$ minutes \\
\hline \multicolumn{3}{|c|}{$\begin{array}{l}\text { BMI = body mass index, COPD = chronic obstructive pulmonary disease, CURB-65 score = confusion, urea, respiratory ra } \\
\text { blood pressure- } \text { score, ARDS }=\text { acute respiratory distress syndrome, } \mathrm{VF}=\text { ventricular fibrillation, } \mathrm{VT}=\text { ventricular tachycar } \\
\mathrm{PEA}=\text { pulseless electric activity, } \mathrm{CPR}=\text { cardiopulmonary resuscitation }\end{array}$} \\
\hline
\end{tabular}




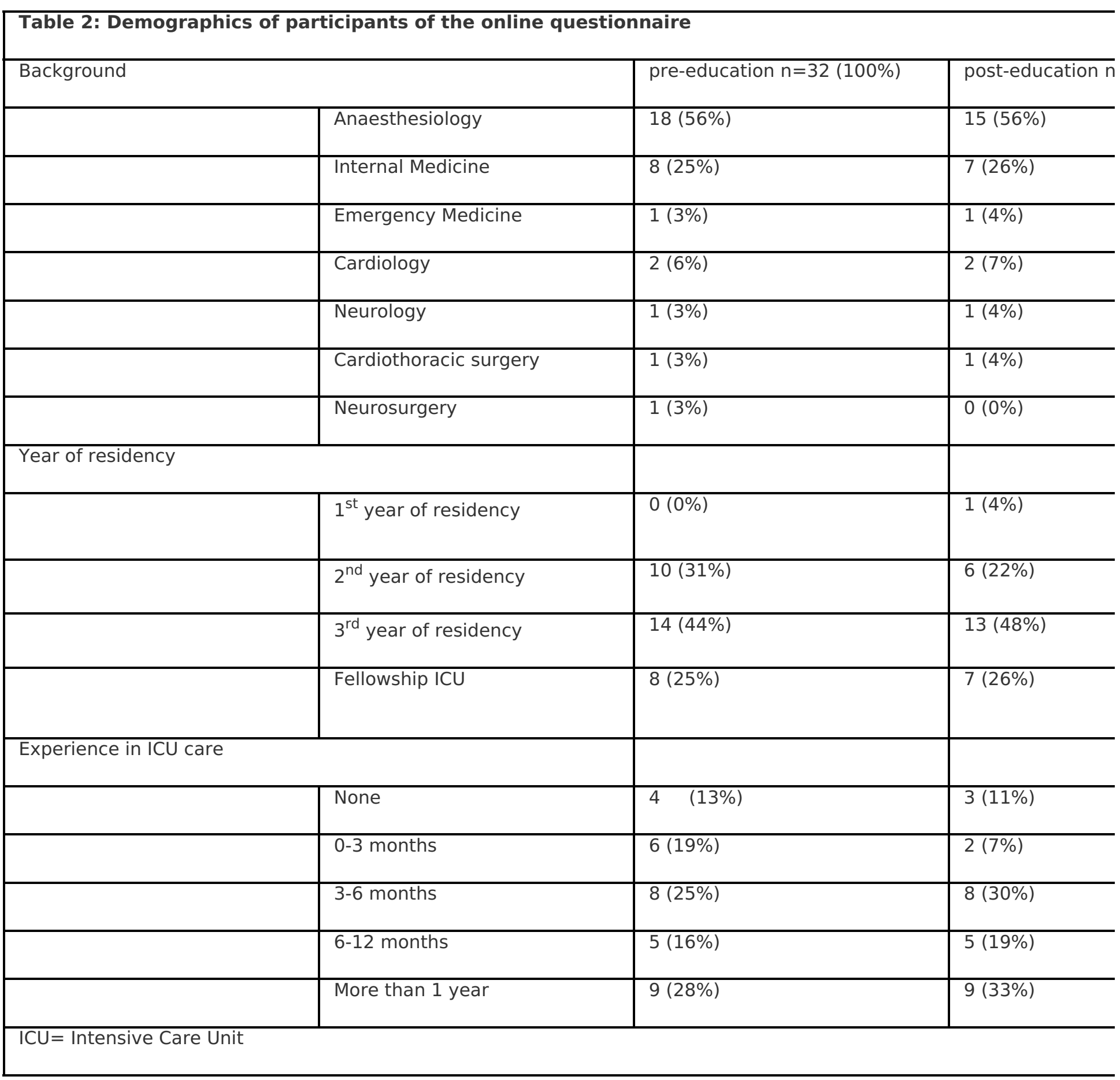




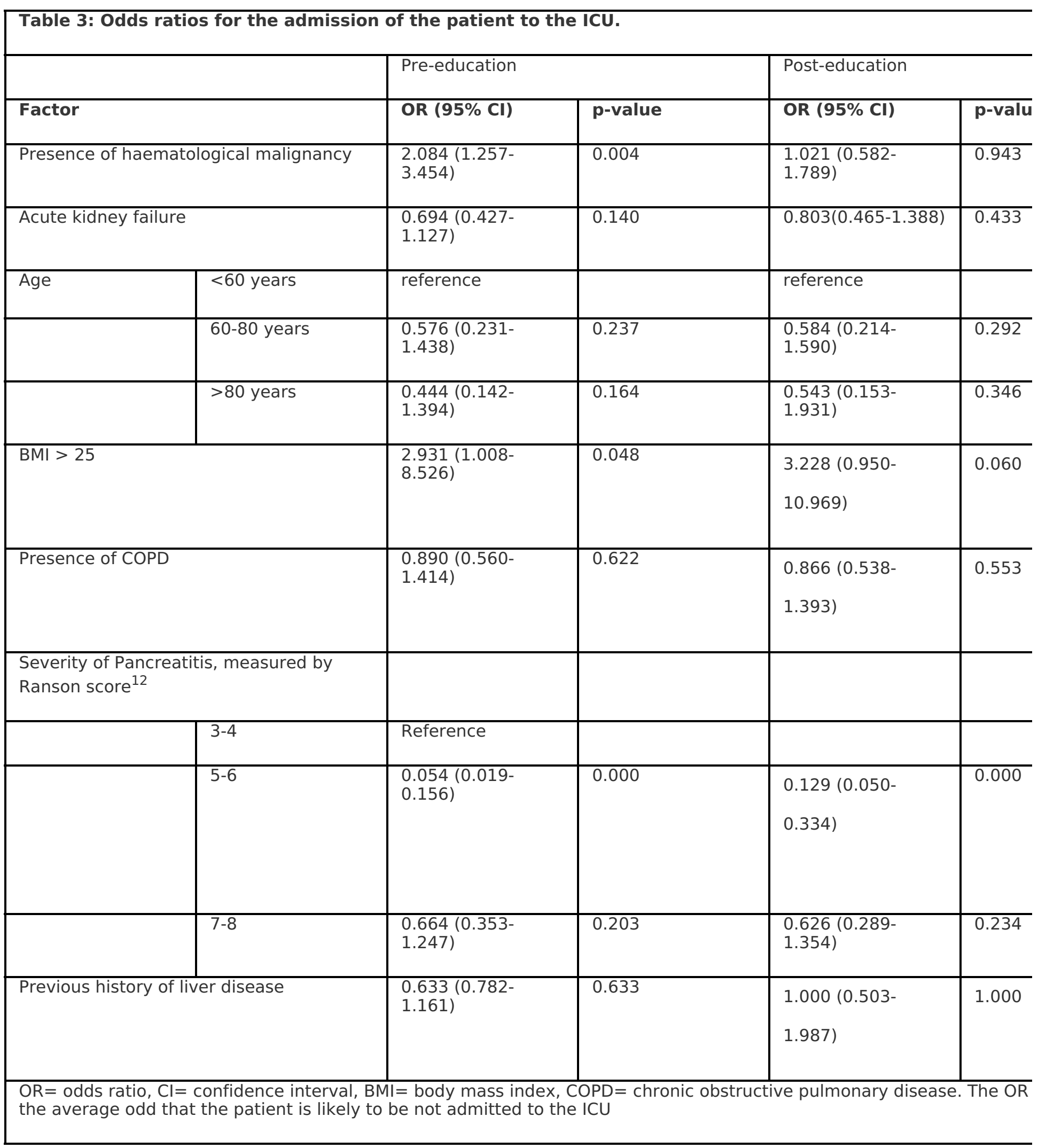


Table 4: Factors of importance in the estimation of mortality in ARDS and cardiac arrest

\begin{tabular}{|c|c|c|c|c|c|c|c|c|}
\hline & \multicolumn{4}{|c|}{ Pre- education } & \multicolumn{4}{|c|}{ Post- education } \\
\hline & \multicolumn{2}{|c|}{ Mortality $40-80 \%$} & \multicolumn{2}{|c|}{ Mortality > 80\% } & \multicolumn{2}{|c|}{ Mortality 40-80\% } & \multicolumn{2}{|c|}{ Mortality > 80\% } \\
\hline Factor & OR (CI) & $\bar{p}$ & OR (CI) & $\mathbf{p}$ & OR (CI) & $\bar{p}$ & OR (CI) & \\
\hline $\begin{array}{l}\text { Presence of } \\
\text { haematolo } \\
\text { gical } \\
\text { malignancy }\end{array}$ & $\begin{array}{l}0.188 \\
(0.042- \\
0.847)\end{array}$ & 0.029 & $\begin{array}{l}0.161 \\
(0.036- \\
0.720)\end{array}$ & 0.017 & $\begin{array}{l}4.320 \\
(2.089- \\
8.933)\end{array}$ & 0.000 & $\begin{array}{l}3.124 \\
(1.497- \\
6.519)\end{array}$ & \\
\hline $\begin{array}{l}\text { Acute } \\
\text { kidney } \\
\text { failure }\end{array}$ & $\begin{array}{l}0.056 \\
(0.007- \\
0.426)\end{array}$ & 0.005 & $\begin{array}{l}0.023 \\
(0.003- \\
0.169)\end{array}$ & 0.000 & $\begin{array}{l}0.79 \\
(0.010- \\
0.604)\end{array}$ & 0.014 & $\begin{array}{l}0.024 \\
(0.003- \\
0.177)\end{array}$ & \\
\hline $\begin{array}{l}\text { Location of } \\
\text { cardiac } \\
\text { arrest }\end{array}$ & $\begin{array}{l}6.740 \\
(0.846- \\
53.708)\end{array}$ & 0.072 & $\begin{array}{l}9.274(1.2- \\
71.697)\end{array}$ & 0.033 & $\overline{\mathrm{NA}}$ & $\overline{\mathrm{NA}}$ & $\begin{array}{l}0.406 \\
(0.168- \\
0.981)\end{array}$ & \\
\hline $\begin{array}{l}\text { Delay until } \\
\text { start CPR }\end{array}$ & $\begin{array}{l}0.045 \\
(0.010- \\
0.199)\end{array}$ & 0.00 & $\begin{array}{l}0.021 \\
(0.005- \\
0.94)\end{array}$ & 0.000 & $\begin{array}{l}0.038 \\
(0.005- \\
0.294)\end{array}$ & 0.02 & $\begin{array}{l}0.013 \\
(0.002- \\
0.100)\end{array}$ & \\
\hline $\begin{array}{l}\text { First heart } \\
\text { rhythm in } \\
\text { cardiac } \\
\text { arrest }\end{array}$ & $\begin{array}{l}0.610 \\
(0.283- \\
1.314)\end{array}$ & 0.207 & $\begin{array}{l}0.631 \\
(0.297- \\
1.343)\end{array}$ & 0.232 & $\begin{array}{l}0.541 \\
(0.269- \\
1.555)\end{array}$ & 0.330 & $\begin{array}{l}0.541 \\
(0.225- \\
1.297)\end{array}$ & \\
\hline $\begin{array}{l}\text { Severity of } \\
\text { ARDS }\end{array}$ & $\begin{array}{l}0.065 \\
(0.018- \\
0.236)\end{array}$ & 0.000 & $\begin{array}{l}0.012 \\
(0.003- \\
0.055)\end{array}$ & 0.000 & $\begin{array}{l}0.056 \\
(0.007- \\
0.443)\end{array}$ & 0.006 & NA & \\
\hline
\end{tabular}

$\mathrm{OR}=$ odds ratio, $\mathrm{Cl}=$ confidence interval $95 \%, \mathrm{CPR}=$ cardiopulmonary resuscitation, $\mathrm{ARDS}=$ acute respiratory distress syr $\mathrm{OHCA}=$ out of hospital cardiac arrest

Figures 

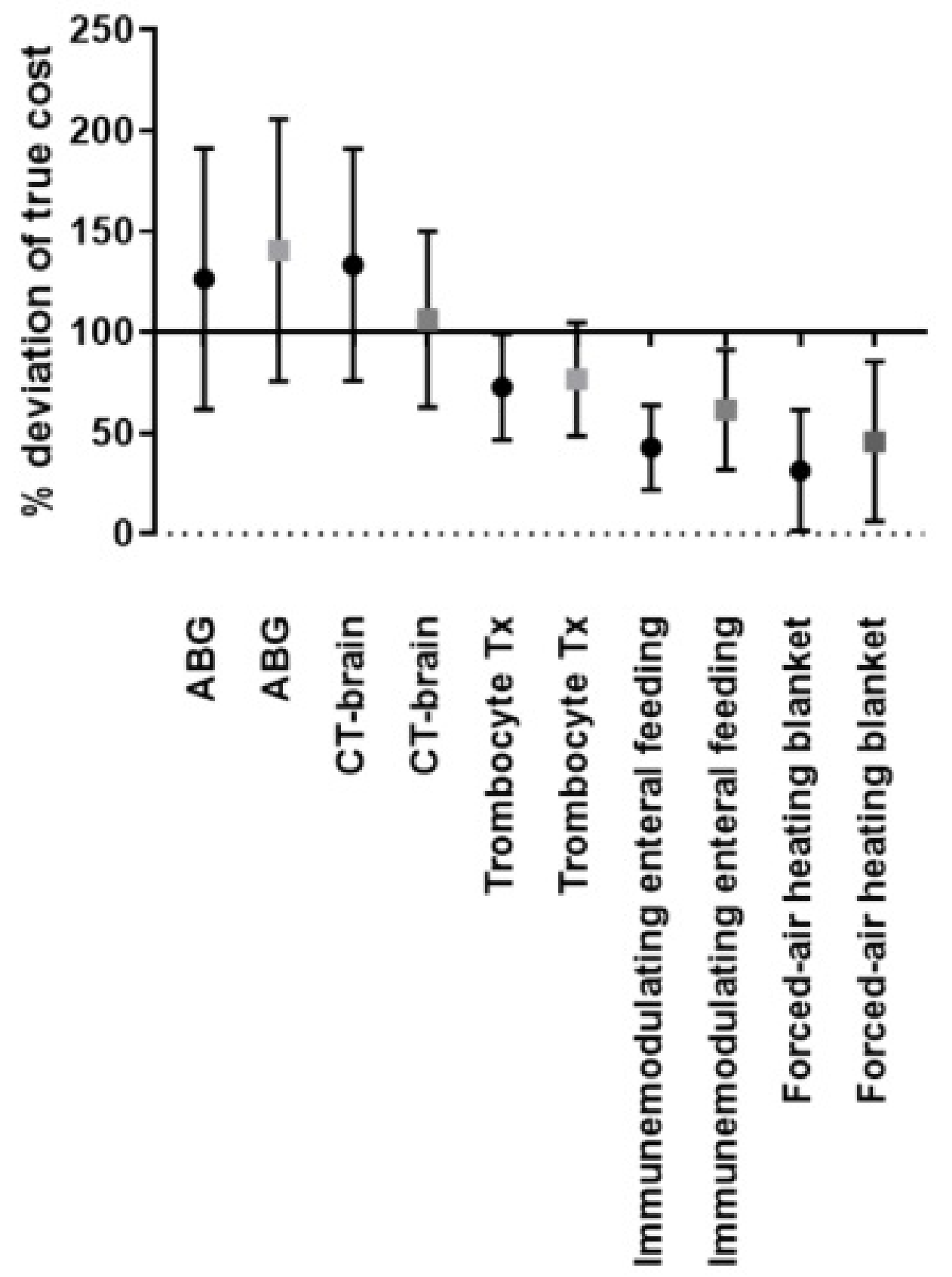

Figure 1

Percentage deviation of true cost of five different ICU products. Dots are pre-education, squares are post-education. Results are shown in means \pm SEM. X-axis at $100 \%$ is the true cost of the product. All values close to the X-axis are approximating true product cost. Abbreviations: $\mathrm{ABG}=$ arterial blood gas analysis, $\mathrm{CT}=$ computed tomography, $\mathrm{Tx}=$ transfusion. 


\section{Supplementary Files}

This is a list of supplementary files associated with this preprint. Click to download.

Supplement 1.pdf

STROBE_checklist_cohort_Wagenberg et al.pdf 